\section{Doubling down on dopamine}

\section{By Lev Osherovich, Senior Writer}

Dopamine receptor antagonists are widely used to treat schizophrenia but can cause metabolic side effects such as weight gain and type 2 diabetes. Now, a Scripps Florida team has pinpointed a receptor complex in the hypothalamus that may play a role in the increase in appetite caused by the drugs. ${ }^{1}$ The challenge now is to design next-generation antipsychotics without the appetite-related side effects.

The receptor complex identified consists of two G protein-coupled receptors-the dopamine $\mathrm{D} 2$ receptor and the ghrelin receptor (GHSR) - that were not previously suspected to interact with one another. Indeed, the D2 receptor until recently was thought to exist as a simple monomer.

GHSR responds to ghrelin, a hormone that regulates appetite.

"From the early days, it was thought that these receptors functioned as single proteins, and a great deal of pharmacology was based on that view," said Graeme Milligan, professor of molecular pharmacology at the University of Glasgow.

However, recent studies by Milligan and others have suggested the D2 receptor can form heterodimers with a variety of other receptors, including the dopamine D3 receptor. ${ }^{2,3}$

"The concept of heterodimeric receptors hasn't received as much attention as it should, perhaps because it's such a new idea," said Daniel Müller, associate professor of psychiatry and head of the pharmacogenomics research clinic at the University of Toronto. Müller's team has identified genetic variants in the D2 receptor and other receptors that influence antipsychotic-induced weight gain. ${ }^{4}$

Milligan said much of the data on heterodimer receptors have come from cell culture models rather than animals, and as a result the physiological significance of these complexes has been unclear.

"These dimeric receptors generate distinctive signals, but so far there hasn't been much evidence of their effect on behavior," said Milligan. "Companies are being very careful about dipping their toes into this area."

Now, a team led by Roy Smith, chairman and professor of metabolism and aging at Scripps Florida, has evidence that such dimers do have a therapeutically relevant function. The team used cell culture and mouse studies to show that a D2 receptor-GHSR heterodimer works in the hypothalamus to regulate appetite.

\section{Complex receptor}

Smith said his team's work stems from prior studies of interactions between GHSR and dopamine D1 receptor.

"Several years ago we reported that dopamine receptor D1 and ghrelin receptor are coexpressed" in the same neurons, said Smith. "We showed that those receptors can form heterodimers, but there was skepticism whether these heterodimers had any physiological function in vivo. We really had to demonstrate that dopamine receptor heterodimers are formed in native tissue."

The D1 receptor is widely expressed in the brain and is not thought to be directly involved in schizophrenia, although it plays a role in other neurological disorders including Parkinson's disease (PD). ${ }^{5}$

In the new work, Smith's team tested whether murine Ghsr and D2 receptor could interact in hypothalamic neurons. The team used in situ fluorescence methods to show that the two receptors colocalized in hypothalamic neurons in brain slices and physically interacted when transfected together in cell culture.

The team then showed that Ghsr increases the effect of agonizing the D2 receptor. Hypothalamic neurons transfected with both the D2 receptor and Ghsr had a stronger response to a D2 receptor agonist than cells transfected with only the D2 receptor. Pharmacological blockade of Ghsr decreased the effectiveness of D2 receptor agonists compared with no blockade.

The team next tested the physiological significance of the D2 receptor-Ghsr complex. Wild-type animals treated with a D2 receptor agonist had lower food intake than vehicle-treated controls. However, in Ghsr knockouts or wild-type mice treated with a Ghsr antagonist, the D2 receptor agonist did not decrease appetite compared with that seen in wild-type mice treated only with the $\mathrm{D} 2$ receptor agonist.

Results were published in Neuron.

\section{Compound pharmacology}

The findings suggest that if a D2 receptor-GHSR complex exists in humans, it is likely to mediate the effects of dopamine on appetite.

Smith, Milligan and Müller agreed that the next step is to identify compounds that selectively block the D2 receptor-GHSR complex and other D2 receptor-containing heterodimers. But the three researchers had different ideas about what kind of compounds would be most therapeutically useful.

"In the long term, if indeed there are ghrelin receptor heterodimers with D1 receptor and D2 receptor in humans, we could specifically design antagonists to target them," said Smith. "We are submitting a grant proposal to screen for compounds that selectively antagonize these complexes."

Smith wants to use such compounds to test whether GHSR-D1 


\section{ANALYSIS}

\section{TARGETS \& MECHANISMS}

receptor or GHSR-D2 receptor dimers play a role in neurological diseases.

Milligan suspects antagonizing the D2 receptor-GHSR complex is likely to elicit excessive appetite, which would not be desirable for therapeutics. He thinks it would be best to avoid hitting GHSR-containing receptors altogether and instead hit other forms of D2 receptor, such as the $\mathrm{D} 2$ receptor-D3 receptor complex that his team identified.

"All conventional antipsychotics are antagonists of D2 receptor, but many antipsychotics are just as effective at the D3 receptor," said Milligan. "You could overcome some of the side effects of these drugs by getting a selective compound for the [D3 receptor-containing] heteromeric complex."

Milligan is collaborating with Servier to test the company's preclinical dopamine receptor antagonists for specific activity against the $\mathrm{D} 2$ receptor-D3 receptor complex his team discovered.

Finally, Müller thinks the best bet could be a partial or inverse agonist of D2 receptor-GHSR that would be antagonistic with respect to psychosis but neutral or perhaps agonistic with respect to the receptor complex's role in suppressing appetite. "D2 receptor has a conflict of interest," he said. "If you agonize it, it blocks weight gain, but this could induce psychosis. It may be the heterodimeric D2 receptor-GHSR could be agonized without taking away the effect of antipsychotics."

Another open question is whether the D2 receptor-GHSR complex plays a role in the broader metabolic effects of ghrelin.

Peter DiStefano, CSO of Elixir Pharmaceuticals Inc., noted that the hypothalamus integrates signals from multiple hormonal pathways involved in sensing energy levels and stimulating appetite. Known metabolic players in the hypothalamus include the peptide hormones ghrelin, orexin, leptin and proopiomelanocortin (POMC), but until now dopamine, which is a small molecule, has not been considered a prime target in metabolism.

"I'd like to see a lot more evidence that targeting dopaminergic signaling" can affect body weight, said DiStefano. He suggested a side-by-side comparison in mice of dopamine receptor agonists and GHSR antagonists on food intake and long-term body weight.

Elixir has GHSR antagonists in preclinical development for obesity and metabolic syndrome and a GHSR agonist that has completed preclinical testing for postoperative ileus and diabetic gastroparesis.

Results of the Neuron study are not patented.

Osherovich, L. SciBX 5(6); doi:10.1038/scibx.2012.143

Published online Feb. 9, 2012

\section{REFERENCES}

1. Kern, A. et al. Neuron; published online Jan. 26, 2012; doi:10.1016/j.neuron.2011.10.038

Contact: Roy G. Smith, Scripps Florida, Jupiter, Fla. e-mail: rgsmith@scripps.edu

2. Perreault, M.L. et al. CNS Neurosci. Ther. 17, 52-57 (2011)

3. Pou, C. et al. J. Biol. Chem.; published online Jan. 30, 2012; doi:10.1074/jbc.M111.326678

4. Lett, T.A.P. et al. Mol. Psychiatry; published online Sept. 6, 2011; doi:10.1038/mp.2011.109

5. Jiang, H. et al. Mol. Endocrinol. 20, 1772-1785 (2006)

COMPANIES AND INSTITUTIONS MENTIONED

Elixir Pharmaceuticals Inc., Cambridge, Mass.

Scripps Florida, Jupiter, Fla.

Servier, Neuilly-sur-Seine, France

University of Glasgow, Glasgow, U.K.

University of Toronto, Toronto, Ontario, Canada 